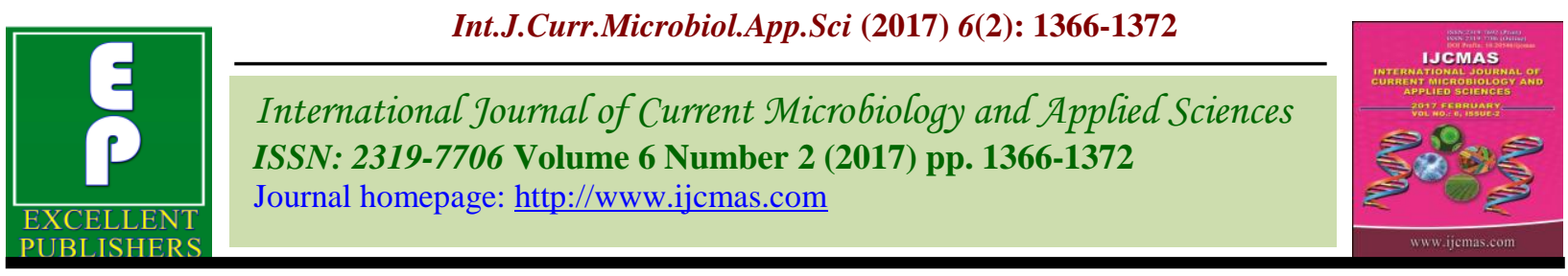

Original Research Article

http://dx.doi.org/10.20546/ijcmas.2017.602.155

\title{
Isolation and Identification of Anaerobic Gram Negative Bacilli In Chronic Periodontitis
}

\author{
Kavitha Karur $^{1 *}$, V.A. Indumathi ${ }^{1}$ and S. Sowmya ${ }^{2}$ \\ ${ }^{1}$ Department of Microbiology, M S Ramaiah Medical College, MSRIT Post, \\ Mathikere, Bangalore-560054, Karnataka, India \\ ${ }^{2}$ Department of Periodontics, Sri Siddhartha Dental College, Agalkote, \\ Tumakuru-572107, Karnataka, India \\ *Corresponding author
}

A B S T R A C T

Periodontitis is a chronic inflammation of the periodontium which progresses to bone resorption and tooth loss in the event of delayed or lack of treatment. Chronic periodontitis is recognized as the most frequently occurring form of periodontitis which is mainly attributed to the presence of Gram negative anaerobic organisms in the sub gingival plaque of these patients. However there are wide differences in the proportion of type of microorganisms in different geographical areas attributed to ethnicity, dietary habits and socio economic status. So the study of sub gingival microbiota in a particular country becomes relevant for understanding their implications in the pathogenesis of periodontal disease and also to identify their possible impact on treatment outcomes. As there is a paucity of information on the prevalence of Gram negative anaerobic micro organisms in our population, the present study was under taken to isolate and identify the Gram negative anaerobic bacilli. This cross sectional study was carried out between January and December 2013 on 71 chronic periodontitis and 55 healthy subjects attending the dental outpatient department of our tertiary care hospital. The sub gingival plaque samples were processed using anaerobic culture to isolate obligate anaerobic Gram negative bacilli. Identification was performed using special potency antimicrobial discs and standard biochemical reactions. $71.84 \%$ in chronic periodontitis and $50.91 \%$ in healthy participants showed the presence of obligate anaerobic Gram negative bacilli. Porphyromonas (13.18\%), Prevotella (32.97\%), Bacteroides (24.17\%) and Fusobacterium (29.67\%) were isolated in chronic periodontitis while Porphyromonas (8.69\%), Prevotella (30.43\%), Bacteroides (26.08\%) and Fusobacterium (34.78\%) were isolated from the control group. The proportion of these putative pathogens was found to be higher in chronic periodontitis patients when compared to healthy subjects. The presence of anaerobic Gram negative bacilli in both study groups points towards the possibility of an opportunistic role that these organisms assume in the presence of risk factors.

\section{Introduction}

Periodontitis is an infectious disease resulting in inflammation within the supporting tissues of the teeth leading to progressive loss of periodontal attachment and terminating with loss of tooth. Chronic periodontitis is recognised as the most frequently occurring form of periodontitis in adults. Even though the aetiology of this disease is multi factorial, 
the presence of Gram negative anaerobic bacilli in the sub gingival niche of the oral cavity is mainly implicated in the pathogenesis of the disease (Mane et al., 2009). Though a large number of bacteria reside in the oral cavity only a few of them are said to be associated with the actual disease initiation and progression. The periodontal pathogens namely Porphyromonas gingivalis, Prevotella intermedia, Actinobacillus actinomycetemcomitans, Fusobacterium nucleatum, Bacteroides forsythus, Capnocytophaga, Selenomonas species are among the bacteria mostly associated with the disease (Mane et al., 2009; Raouf et al., 1994).

Various studies around the world have found a common pattern in the presence of potential pathogenic microorganisms in the sub gingival plaque but there is a difference which exists in their prevalence which may be attributed to ethnic differences, dietary habits, use of over the counter antimicrobial drugs, development level and sanitation conditions (Lafaurie et al., 2007). The recent emphasis on studying the pathogenic role of periodontal pathogens in the disease is also because of the proposed modulating role of periodontal disease in cardiovascular and cerebro vascular disease, diabetes and respiratory disease and adverse pregnancies. The inflammatory mechanisms active in the periodontal disease has the potential to play a pathogenic role in some of the conditions studied like Rheumatoid Arthritis and Alzheimer's disease (Lalitha, 2011; Angela et al., 2008).

Hence the study of subgingival microbiota in a particular community becomes relevant for understanding their implications in the pathogenesis of periodontal disease and to identify their possible impact on outcomes after treatment. As there is a paucity of information on the prevalence of Gram negative anaerobic micro organisms in our population, the present study was under taken to isolate and identify the Gram negative anaerobic bacilli.

\section{Materials and Methods}

\section{Study population}

The study subjects were selected from the outpatient department of periodontics of M.S. Ramaiah Dental College and Hospital, Bangalore, Karnataka.

The study population included 71 chronic periodontitis patients with periodontal pocket depth $\geq 5 \mathrm{~mm}$ and CAL of $>3 \mathrm{~mm}$. 55 healthy subjects without the clinical signs of periodontal disease were also selected. The study subjects were in the age range of 25- 60 years.

Patients with any systemic disease, pregnant and lactating mothers, who have taken systemic antimicrobials or any antiinflammatory drugs in the previous 6 months and who have undergone any kind of periodontal therapy six months prior to initial examination were excluded from the study.

After Institutional Ethical Committee approval, the purpose and the nature of the study was explained to the subjects and consent for the participation in the study was obtained prior to the commencement of the study.

\section{Specimen collection}

For microbiological evaluation sample were collected from the deepest four pockets in chronic periodontitis patients where as samples from four different sites was taken for periodontally healthy subjects. Under aseptic precautions microbial samples were taken from the participants using sterile Gracey curettes and then transferred to vials containing $2 \mathrm{ml}$ of thioglycollate medium 
enriched with vitamin $\mathrm{k}$ and hemin and transported to the laboratory within 1 hour of collection for further processing.

\section{Anaerobic culture of specimen}

The specimen received were studied with Gram stain and sub cultured on the following media to isolate obligate anaerobic gram negative bacilli.

Non selective medium: Brucella blood agar (BBA) (with 5\% sheep blood) supplemented with vitamin $\mathrm{K}$ and hemin.

Special media like kanamycin blood agar for selective isolation of Porphyromonas species, kanamycin and vancomycin blood agar for selective isolation of Prevotella species and neomycin and vancomycin blood agar for selective isolation of Fusobacterium species (Saini et al., 2003).

These plates were incubated in an anaerobic jar with anaerobic gas generating system sachet with indicator (BD Gaspak ${ }^{\mathrm{TM}}$ EZ Anaerobe Container System Sachets With Indicator Code 260001) at 35 to $37^{\circ} \mathrm{C}$ for 48 -72 hours.

The colonies on primary plates were tested for aero tolerance on chocolate agar plates. Gram negative bacilli identified by colony morphology and gram stain were processed further. Identification was based on susceptibility patterns to special potency antimicrobial discs of kanamycin $(1000 \mathrm{mcg})$, vancomycin $(5 \mathrm{mcg})$ and colistin $(10 \mathrm{mcg})$ and standard biochemical tests like spot indole, catalase, nitrate reduction and sugar fermentation tests.

\section{Results and Discussion}

The colony count was expressed in mean with standard deviation. The culture of the dental plaque samples yielded growth of anaerobic Gram negative bacilli in $71.84 \%$ (51) of the chronic periodontitis patients and in $50.91 \%$ (28) of healthy controls.

Among the chronic periodontitis patients $70.58 \%$ (36) showed the growth of multiple anaerobic gram negative bacilli compared to $29.42 \%$ (15) that showed single type. 3 types of bacterial isolates were recovered in 9 cases $(18 \%)$ and 2 , in 18 (36\%). In the control group $42.85 \%$ (12) participants showed multiple anaerobic gram negative bacilli in culture.

\section{Proportion of anaerobic Gram negative bacilli}

Out of 91 isolates, Porphyromonas species constituted $13.18 \%$ (12), Prevotella species $32.97 \%$ (30), Fusobacterium species $29.67 \%$ (27) and Bacteroides fragilis group $24.17 \%$ (22) of the total anaerobic gram negative isolates in chronic periodontitis patients. In healthy controls a total of 46 isolates were recovered. Among them Porphyromonas species constituted $8.69 \%$ (4), Prevotella species $30.43 \%$ (14), Fusobacterium species $34.78 \%$ (16) and Bacteroides fragilis group $26.08 \%$ (12) (Table 1 and 2).

In the present study $71.84 \%$ of chronic periodontitis subjects and $50.91 \%$ of healthy participants showed the growth of obligate anaerobic Gram negative bacilli, which falls within the wide range of 42 to $100 \%$ noted in several studies conducted in India and abroad. This variation in the recovery rate may be attributed to variables in criteria of patient selection, culture method employed, geographical differences and use of molecular techniques for identification. Periodontitis is usually a polymicrobial disease. In the present study poly microbial isolation of anaerobic Gram negative bacilli was found in $70.42 \%$ of cases with 9 participants showing three types 
and 18 showing two types of obligate anaerobic Gram negative bacilli. 29.58\% showed growth of a single type of obligate anaerobic Gram negative bacillus along with presence of Gram positive bacteria. The poly microbial growth in healthy participants was $42.85 \%$. Similar to our study poly microbial growth of $72.28 \%$ and $100 \%$ of chronic periodontitis was reported in studies by Mane et al., (2009) and Saini (2003).

The results of our study show that there is a complex ecological relationship of the sub gingival flora in periodontitis which is determined chiefly by the presence of anaerobic Gram negative bacilli. Porphyromonas species constituted $13.18 \%$ of the total isolates obtained with mean colony counts of $72.91 \pm 19.82 \times 10^{3} \mathrm{cfu} / \mathrm{ml}$ in chronic periodontitis patients and $8.69 \%$ with mean colony count of $13 \pm 8.21 \times 10^{3}$ in healthy participants (Graph 1). Kumar et al., (2006) Griffin et al., (1998) have shown that Porphyromonas is highly associated with periodontitis in their studies.

However, there are studies like that of Birgit et al., (2009) that did not find statistically significant difference in the isolation of Porphyromonas in periodontitis and healthy group, contrary to the popular belief. According to the / these authors, isolation of Porphyromonas is better correlated with periodontal pocket depth than the disease definition.

Prevotella constituted $32.97 \%$ of the isolates with a mean colony count of $68.17 \pm 16.53 x$ $10^{3} \mathrm{cfu} / \mathrm{ml}$ in chronic periodontitis and $30.43 \%$ with mean colony counts of $33.46 \pm$ $9.21 \times 10^{3} \mathrm{cfu} / \mathrm{ml}$ in healthy population. It belongs to the orange complex group which appears when the symbiotic host- microbe relationship begins to derail and pathogenic microorganisms begin to establish a strong presence. With further deterioration in the oral hygiene status, orange complex is replaced by the red complex (Sameera et al., 2013). The predominant organism in our study is Prevotella which indicates that the cases of chronic periodontitis have possibly been intercepted in the early phases of the disease. Piret Koll-Klais et al., (2005), also reports Prevotella species (73\%) as the most frequently isolated species in their study.

In the present study Fusobacterium was isolated in $29.67 \%$ with mean colony count of $27.29 \pm 8.32 \times 10^{3} \mathrm{cfu} / \mathrm{ml}$ in chronic periodontitis patients and $34.78 \%$ with mean colony count of $5.63 \pm 10^{3} \mathrm{cfu} / \mathrm{ml}$ in healthy population. Fusobacterium is a prominent component and is one of the first Gramnegative species to establish in plaque biofilms. It is a central species in physical interactions between Gram-positive and Gram-negative species that are likely to be important in bio film colonization, and contributes to the reducing conditions necessary for the emergence of oxygenintolerant anaerobes. It is considered as an intermediate colonizer bridging the attachment of commensal that colonize the tooth and epithelial surface with true pathogens.

In the present study Bacteroides fragilis species was isolated in $24.17 \%$ with mean colony counts of $16.27 \pm 7.76 \times 10^{3} \mathrm{cfu} / \mathrm{ml}$ in chronic periodontitis and in $26.08 \%$ with mean colony counts of $4.08 \pm 2.10 \times 10^{3}$ $\mathrm{cfu} / \mathrm{ml}$ in healthy participants. Contrasting our study, (Mahalakshmi et al., 2012), Porphyromonas was isolated in $80.50 \%$ of cases and $11 \%$ of healthy controls showing that the role of this red complex organism is crucial in disease progress. Prevotella was isolated in $29.70 \%$ and $21.50 \%$ of chronic periodontitis cases and healthy controls respectively.

Mohammed et al., (2004) studied the role of cultivable bacteria in chronic periodontitis and isolated Prevotella in $15.4 \%$, 
Porphyromonas in $21.9 \%$ and Fusobacterium in $0.4 \%$ of chronic periodontitis participants. Contrasting our results, this study found multi bacterial growth in $18.2 \%$ participants.

Mane et al., (2009) studied the periodontal flora in healthy and periodontitis cases and reported Porphyromonas in $48 \%$, Fusobacterium 24\%, and Prevotella spp. in $26 \%$ of the anaerobes isolated in periodontitis patients making them the commonest species in disease. Bacteroides species was reported to be isolated in $5 \%$ cases. Study by Saini et al., (2003) points to the increase in the number of anaerobes in periodontitis patients, thereby reducing the ratio of aerobes and anaerobes to 0.56 . They found that poly microbial growth was seen in all periodontitis participants and Porphyromonas was the commonest isolate along with Prevotella and Fusobacterium.

Table.1 Identification based on special potency antibiotic discs

\begin{tabular}{|l|c|c|c|}
\hline Organisms & Kanamycin $1000 \mu \mathrm{g}$ & Vancomycin $5 \mu \mathrm{g}$ & Colistin $10 \mu \mathrm{g}$ \\
\hline B.fragilis group & $\mathrm{R}$ & $\mathrm{R}$ & $\mathrm{R}$ \\
\hline Porphyomonas & $\mathrm{R}$ & $\mathrm{S}$ & $\mathrm{R}$ \\
\hline Prevotella & $\mathrm{R}$ & $\mathrm{R}$ & $\mathrm{V}$ \\
\hline Fusobacterium & $\mathrm{S}$ & $\mathrm{R}$ & $\mathrm{S}$ \\
\hline
\end{tabular}

Table.2 Comparison of organisms isolated in cases and controls

\begin{tabular}{|lll|} 
Organism & case & Control \\
\hline Porphyromonas species & $12(13.18 \%)$ & $4(8.69 \%)$ \\
\hline Prev otella species & $30(32.97 \%)$ & $14(30.43 \%)$ \\
\hline Fusobacterium species & $27(29.67 \%)$ & $16(34.78 \%)$ \\
\hline Bacteroides fragilis group & $22(24.17 \%)$ & $12(26.08 \%)$ \\
\hline Total isolates & 91 & 46 \\
\hline
\end{tabular}

Graph.1 Representation of mean colony counts

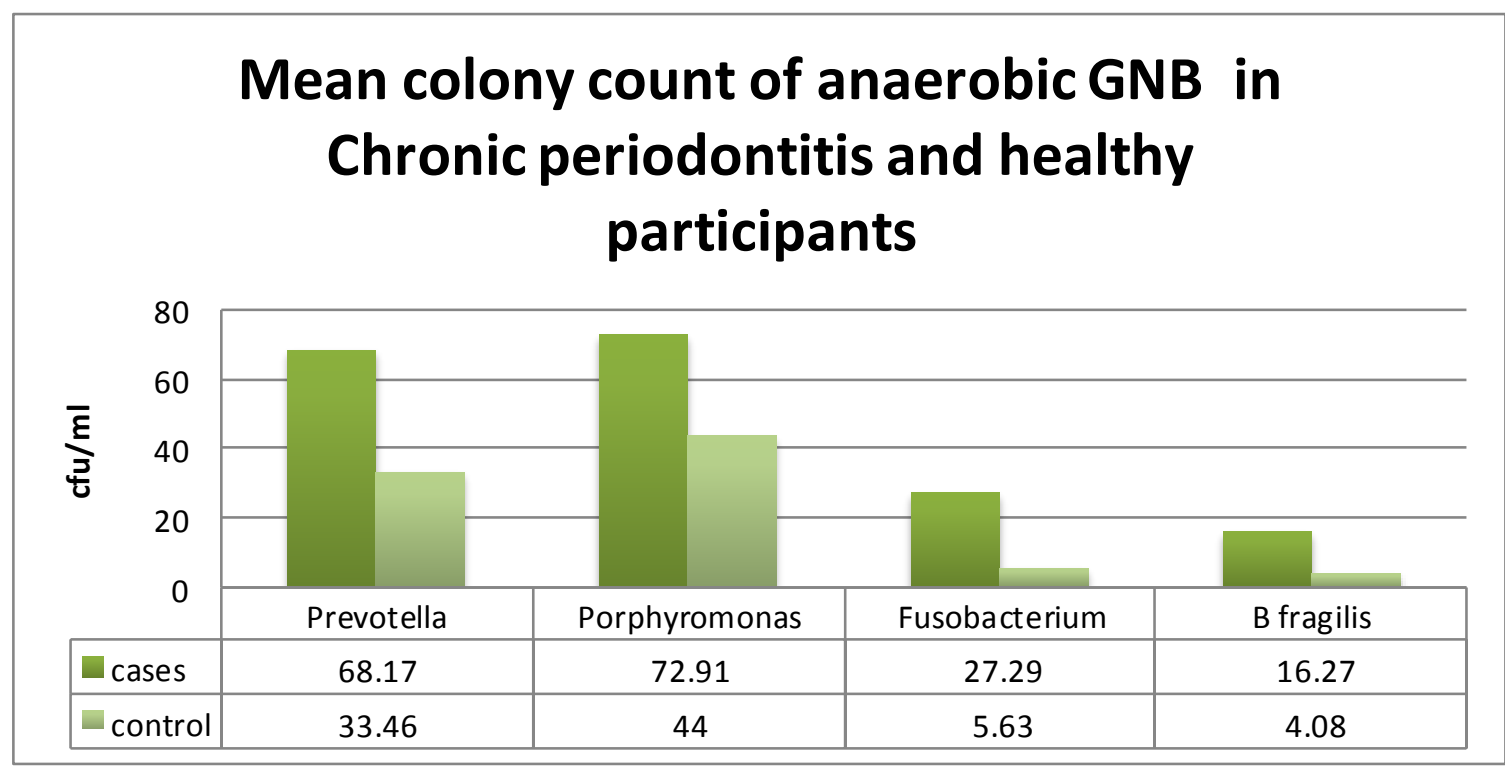


The advent of molecular techniques has led to the expansion of the list of key periodontal pathogens involved in periodontal disease. The role of anaerobic Gram negative bacteria as the sole periodontal pathogens stands seriously challenged with the discovery of many new and uncultivable bacterial species including many viruses, capnophilic and Gram positive bacteria in the sub gingival plaques in a higher proportion.

In conclusion, the isolate in highest proportion in chronic periodontitis patients in our study was Prevotella species followed by Fusobacterium and Bacteroides fragilis group. This study serves as a baseline data for the oral flora prevalent in our population for future studies and interventions.

Although culture methods stands out as 'Gold standard' the use of traditional culture methods limits the isolation to only cultivable bacteria thereby pushing the other non cultivable and hitherto unidentified potential pathogens into oblivion. Hence further study using other advanced molecular techniques on larger sample size in chronic periodontitis as well as in various other forms of periodontitis should be carried out.

\section{References}

Angela, R., Kamer, Ronald, G., Craig Ananda P. 2008. Inflammation And Alzheimer's Disease: Possible Role Of Periodontal Disease. Alzheimer's And Dementia, Elsevier 4: 242-250.

Birgit Riep, Lilian Edesi-Neu, Friderike Claessen. Are Putative Periodontal Pathogens Reliable Diagnostic Markers? J. Clin. Microbiol., P. 17051711.

Griffen, A.L., M.R. Becker, S.R. Lyons, M.L. Moeschberger, and E.J. Leys. 1998. Prevalence of Porphyromonas gingivalis and periodontal health status.
J. Clin. Microbiol., 36: 3239-3242.

Herrera, D., Contreras, A., Gamonal, J., et al., 2008. Subgingival microbial profiles in chronic periodontitis patients from Chile, Colombia and Spain. J. Clin. Periodontol., 35: 106-113.

Kolenbrander, P.E. 2000. Oral microbial communities: biofilms, interactions, and genetic systems. Annu. Rev. Microbiol., 54: 413-37.

Kolenbrander, P.E., Andersen, R.N., Blehert, D.S., Egland, P.G., Foster, J.S., Palmer, R.J. Jr. 2002. Communication among oral bacteria. Microbiol. Mol. Biol. Rev., 486-505.

Krishnan Mahalakshmi, Padma Krishnan, S.C. Chandrasekaran, K.H. Panishankar, Natarajan Subashini. 2012. Prevalence of Periodontopathic Bacteria in the Subgingival Plaque of a South Indian Population with Periodontitis, $J$. Clin. Diag. Res., Vol 6 issue 4: 747752.

Kumar, P.S., E.J. Leys, J.M. Bryk, F.J. Martinez, M.L. Moeschberger, and A. L. Griffen. 2006. Changes in periodontal health status are associated with bacterial community shifts as assessed by quantitative $16 \mathrm{~S}$ cloning and sequencing. J. Clin. Microbiol., 13: 3665-3673.

Lafaurie, G.I., Contreras, A., Baro, A., Botero, J., Fayad, Jaramillo, A., et al., 2007. Demographic, Clinical, and Microbial Aspects of Chronic and Aggressive Periodontitis in Colombia: A Multicenter Study. J. Periodontol., 78: 629-639.

Lalitha, T.A. 2011. Periodontitis And Rheumatoid Arthritis - The Dual Link. Indian J. Dental Advancements, 3(2): 521-25.

Mane, A.K., Karmarkar, A.P., Bharadwaj, R.S. 2009. Anaerobic Bacteria in Subjects with Chronic Periodontitis and 
In Periodontal Health. J. Oral Health Comm. Dent., 3(3): 49-51.

Mohammad Hossein Salari, Zainab Kadkhoda. 2004. Rate Of Cultivable Subgingival Periodontopathogenic Bacteria In Chronic Periodontitis $J$. Oral Sci., Vol. 46(3):157-161.

Piret ko ll-klais, Mandar, R., Leibur, E., Mikelsaar, M. 2005. Oral microbial ecology in chronic periodontitis and periodontal health. Microbial Ecol. Health and Dis., 17: 146-/155.

Raouf Wahab Ali, Vidar Bakken, Rune Nilsen. 1994. Comparative Detection
Frequency Of 6 Putative Periodontal Pathogens In Sudanese And Norwegian Patients. J. Periodontal., Vol 65(11): 1046-52.

Saini, S., Aparna, N. Gupta, A. Mahajan, D.R. 2003. Arora Microbial Flora In Orodental Infections, Indian J. Med. Microbiol., 21(2):111-114.

Sameera, G., Nath, Ranjith Raveendran. 2013. Microbial dysbiosis in periodontitis $J$. Indian Society of Periodontol., Vol 17, Issue 4.

\section{How to cite this article:}

Kavitha Karur, Indumathi, V.A. and Sowmya, S. 2017. Isolation and Identification of Anaerobic Gram Negative Bacilli In Chronic Periodontitis. Int.J.Curr.Microbiol.App.Sci. 6(2): 1366-1372. doi: http://dx.doi.org/10.20546/ijcmas.2017.602.155 